\title{
Component Alignment in Simultaneous Bilateral versus Unilateral Total Knee Arthroplasty
}

\author{
Irfan Qadir, MBBS, Bahar Shah, MBBS, Muhammad Waqas, FCPS, Umair Ahmad, MBBS, \\ Shahzad Javed, FCPS, and Amer Aziz, FCPS, FRCS \\ Department of Orthopaedic and Spine Surgery, Ghurki Trust Teaching Hospital, Lahore, Pakistan
}

\begin{abstract}
Purpose: The purpose of this study was to investigate differences in component alignment between first and second knees in simultaneous bilateral total knee arthroplasty (SBTKA) and unilateral TKA (UTKA).

Materials and Methods: 274 SBTKAs and 198 UTKAs were included in study. Patients were divided into three groups as SBTKA on the right knee (group A), SBTKA on the left knee (group B) and UTKA (group C). Femoral and tibial component alignment was checked in both coronal plane (alpha $[\alpha]$ and beta $[\beta]$ angles) and sagittal plane (gamma $[\gamma]$ and delta $[\delta]$ angles) radiographs.

Results: There were no statistically significant differences among groups in the preoperative anatomical varus angle and Kellgren-Lawrence gonarthrosis classification grade $(\mathrm{p}=0.139$ and $\mathrm{p}=0.329$, respectively). In the coronal plane, the alignment of femoral component ( $\alpha$ angle) and tibial component ( $\beta$ angle) was similar in all three groups ( $\alpha$ angle, 95.01 vs. 95.14 vs. $94.9, p=0.945 ; \beta$ angle, 90.03 vs. 89.67 vs. 89.98 , $p=0.483$ ). The sagittal plane alignment of femoral component ( $\gamma$ angle) and tibial component ( $\delta$ angle) did not show significant differences ( $\gamma$ angle, 7.04 vs. 6.98 vs. 7.00 , $\mathrm{p}=0.132 ; \delta$ angle, 86.56 vs. 87.41 vs. $86.73, \mathrm{p}=0.610$ ).
\end{abstract}

Conclusions: The angular alignment of components was similar between SBTKA and UTKA.

Keywords: Knee, Arthroplasty, Alignment, Position, Component, Simultaneous bilateral

\section{Introduction}

Degenerative changes due to osteoarthritis commonly affect both hips and knees concurrently, thereby warranting bilateral total knee arthroplasty (TKA). This is reflected by the increasing number of patients undergoing simultaneous bilateral TKA (SBTKA) worldwide ${ }^{1}$. The potential benefits of SBTKA compared with staged procedures include a decreased length of hospitalization, decreased time under anesthesia, decreased rehabilitation time, and decreased cost on the healthcare system ${ }^{1)}$.

Received August 13, 2018; Revised (1st) August 11, 2018;

(2nd) October 4, 2018; Accepted October 31, 2018

Correspondence to: Irfan Qadir, MBBS

Department of Orthopaedic and Spine Surgery, Ghurki Trust Teaching

Hospital, Jallo Mor, Lahore, Pakistan

Tel: +92-300-7321600, Fax: +92-61-6778718

E-mail: irfanqadir88@gmail.com

This is an Open Access article distributed under the terms of the Creative Commons Attribution Non-Commercial License (http://creativecommons.org/licenses/by-nc/4.0/) which permits unrestricted non-commercial use, distribution, and reproduction in any medium, provided the original work is properly cited.
Comparison of SBTKA and unilateral TKA (UTKA) in terms of functional outcome has been extensively conducted in previous reports, with results varying from UTKA producing better outcomes than SBTKA ${ }^{2-4)}$, the 2 procedures producing no significant difference ${ }^{1,3,5-7)}$ to SBTKA producing a better outcome than UTKA $^{8-10}$. There is currently no clear consensus on the benefits of SBTKA.

It has been conjectured that the technical performance of SBTKA would be inferior to that of UTKA because of the increased length of time required for the simultaneous procedure ${ }^{11}$. Component malalignment is one of the common problems in TKA, which may impact the long-term outcome of TKA ${ }^{12}$. Although the results of SBTKA are well substantiated, there is a dearth of data reflecting the differences in technical aspects of surgery between both sides. Seo et al. ${ }^{11)}$ reported that in the second TKA, there was a greater incidence rate of outlier in the mechanical femorotibial angle ( $16.2 \%$ vs. $9.0 \%$ ), more blood loss $(735 \mathrm{~mL}$ vs. $656 \mathrm{~mL}$ ), and longer operation time (61 minutes vs. 58 minutes), as compared to the first TKA, while no significant differences were noted in clinical outcomes. On the contrary, Kilincoglu et 
al. ${ }^{12)}$ reported no statistically significant difference in the component alignment between the SBTKA group and the UTKA group. Therefore, we sought to investigate the difference in component alignment between SBTKA and UTKA cases to ascertain the feasibility of SBTKA from technical aspects of surgery.

\section{Materials and Methods}

A retrospective review of 472 consecutive patients who underwent TKA for bilateral varus osteoarthritic deformity at the knee joint between January 2014 and December 2017 at Ghurki Trust Teaching Hospital was conducted. Patients with inflammatory arthritis, posttraumatic arthritis, and valgus deformity at the knee were excluded from the study as well as those who had previously undergone femoral or tibial osteotomy. The patient data were reviewed for clinico-demographic characteristics. A preoperative assessment was done to determine Kellgren-Lawrence classification grades for gonarthrosis.

\section{Surgical Technique}

All surgeries were done by a single surgeon (AA) in the same sequence. Intravenous antibiotics were given half hour before incision and continued till postoperative day 2. Epidural anesthesia was given to all patients. As per surgeon's preference (who is right-hand dominant), the right knee was operated first in all patients. A posterior cruciate ligament-substituting prosthesis (NexGen Legacy; Zimmer, Warsaw, IN, USA) of a traditional design was used in all cases. After elevation and exsanguination of the limb, the tourniquet was inflated up to $300 \mathrm{mmHg}$. A midline incision was made and the knee joint was exposed using the traditional medial parapatellar approach. An intramedullary femoral guide with $5^{\circ}$ angle was used for the femoral cut in an attempt to restore nearly normal anatomical articulation of the distal femur on the proximal tibia ${ }^{13}$. An extramedullary tibial guide with a perpendicular cut to the tibial anatomical axis was used for the tibial cut. Patellar osteophytes were excised and patellar denervation was performed by circumferential electrocautery ${ }^{14)}$. The same procedure was repeated for the other side after wound closure and dressing of the first operated side. Closed suction drains were kept on each side. Drain outputs were recorded separately and the drains were taken out after 48 hours. Patients were mobilized after drain removal.

\section{Radiographic Evaluation}

Radiographs of operated knees were obtained in full extension: an anteroposterior (AP) view with the patella centered over the femoral condyles and a lateral view with superimposed femoral condyles. Both epicondyles should be at the right angle to the radiological cassette. For the true lateral view of the proximal tibia, the tibial tubercle should be located anteriorly and the fibular head should be palpated at the back of the tibial plateau.

All preoperative and postoperative radiographs were evaluated by the same method. The patient X-rays were divided for evaluation as SBTKA on the right knee (group A), SBTKA on the left knee (group B), and UTKA (group C). The preoperative assessment was done according to the Kellgren-Lawrence classification. The anatomical femoral axes and anatomical tibial axes were drawn in preoperative standing AP radiographs to measure the varus angle. Alpha angle was defined the medial angle between a line drawn parallel to the femoral component condyles and the anatomical axis of the femur. Beta angle was measured as the medial angle between a line drawn parallel to the tibial component on the AP X-ray and the anatomical axis of the tibia. Sagittal femoral gamma angle was determined as the proximal angle between a line drawn perpendicular to the distal cement interface of the femoral component and the femoral anatomical axis on the lateral X-ray. Sagittal tibial delta angle was the posterior angle between a line drawn parallel to the tibial component and the anatomical tibial axis on the lateral X-ray (Fig. 1). Components outside the conventional $\pm 3^{\circ}$ range from the neutral alignment of $90^{\circ}$ in the coronal plane were considered outliers for component alignment. All digital radiographic images were analyzed using ImageJ ver. 1.51 (National Institutes of Health, Bethesda, MD, USA). Two independent investigators (BS and MW) measured

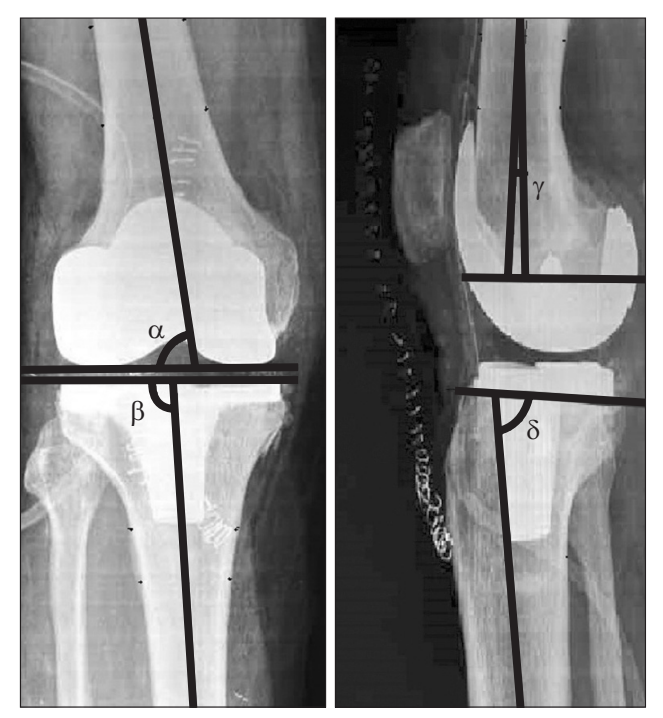

Fig. 1. Radiographic measurement of component position using alpha $(\alpha)$, beta $(\beta)$, gamma $(\gamma)$, and delta $(\delta)$ angles. 
these angles on preoperative and postoperative radiographs. Intraclass correlation coefficients (ICC) were used to assess the degree of agreement within a rater or between raters. The ICC for intra-observer variability was 0.88 , and ICC for inter-observer variability was 0.83 . There was good or excellent inter-observer agreement in all of the measurements performed.

\section{Statistical Analysis}

Patient data and the preoperative and postoperative radiographic alignment data are expressed as mean and standard deviation. The Kruskal-Wallis test was used for the comparison of Kellgren-Lawrence classification grades, preoperative anatomical varus angles and postoperative alpha, beta, gamma and delta angles of the three groups (groups A, B, and C). The statistical analyses were done using IBM SPSS ver. 20 (IBM Corp., Armonk, NY, USA).

\section{Results}

There were 286 females and 186 males with a mean age of 64.3 \pm 5.6 years. Among them, 274 and 198 patients underwent SBTKA and UTKA, respectively. Most of the patients belonged to Kellgren-Lawrence grades 3 and 4 and the difference in Kellgren-Lawrence grading and preoperative varus angle between groups was not significant ( $\mathrm{p}=0.329$ and $\mathrm{p}=0.139$, respectively). The mean postoperatively measured alpha, beta, gamma, and delta angles of the three groups (groups A, B, and C) are given in Table 1 . There were no statistically significant differences in the postoperatively measured alpha, beta, gamma, and delta angles of the three groups $(\mathrm{p}=0.945, \mathrm{p}=0.483, \mathrm{p}=0.132$, and $\mathrm{p}=0.610$, respectively) (Table 1 ). The incidence of outliers of the femoral components was $67.0 \%, 70.3 \%$, and $59.6 \%$ in group A, B, and C, respectively. The incidence of outliers of the tibial components was $14.3 \%, 12.1 \%$, and $12.7 \%$ in group $\mathrm{A}, \mathrm{B}$, and $\mathrm{C}$, respectively.
However, no significant differences were observed in outlier rates of the femoral and tibial components between groups $(\mathrm{p}=0.445$ and $\mathrm{p}=0.905$, respectively) (Table 2 ).

\section{Discussion}

The results of our study indicate that there were no significant differences in component alignment between SBTKA and UTKA. The incidence of outliers of the femoral component was erroneously high but similar between SBTKA and UTKA. The incidence of outliers of the tibial component was similar between study groups and on par with published literature. Despite the retrospective design, our study is strengthened by the homogeneity of cases in all subgroups.

The potential benefits of SBTKA compared with staged procedures include a decreased length of hospitalization, decreased time under anesthesia, decreased rehabilitation time, and decreased cost on the healthcare system ${ }^{1)}$. Proponents of SBTKA argue that there is no difference in component alignment between SBTKA and UTKA. Furthermore, the first operation can provide information and aid the surgeon conducting the later contralateral side operation ${ }^{11,15)}$. However, from a developing nation's per-

Table 2. Post Hoc Analysis for Pairwise Comparison Groups

\begin{tabular}{lccc}
\hline \multirow{2}{*}{ Variable } & \multicolumn{3}{c}{ Group } \\
\cline { 2 - 4 } & A vs. B & A vs. C & B vs. C \\
\hline Alpha angle & 0.888 & 0.931 & 0.712 \\
Beta angle & 0.259 & 0.978 & 0.430 \\
Gamma angle & 0.881 & 0.954 & 0.988 \\
Delta angle & 0.019 & 0.874 & 0.119 \\
\hline
\end{tabular}

p-values according to Tukey's honestly significant difference test. Group A: simultaneous bilateral total knee arthroplasty (SBTKA) on the right knee, Group B: SBTKA on the left knee, Group C: unilateral TKA.

Table 1. Statistical Comparison of Study Variables among Groups

\begin{tabular}{|c|c|c|c|c|}
\hline Variable & Group A & Group B & Group C & p-value \\
\hline Varus angle $\left({ }^{\circ}\right)$ & $7.2 \pm 0.7$ & $7.1 \pm 0.7$ & $7.3 \pm 0.7$ & 0.139 \\
\hline Alpha angle $\left(^{\circ}\right)$ & $95.0 \pm 3.5$ & $95.1 \pm 2.7$ & $94.9 \pm 3.7$ & 0.945 \\
\hline Beta angle $\left({ }^{\circ}\right)$ & $90.0 \pm 3.0$ & $89.7 \pm 2.4$ & $90.0 \pm 2.7$ & 0.483 \\
\hline Gamma angle $\left(^{\circ}\right)$ & $7.0 \pm 1.0$ & $7.0 \pm 0.8$ & $7.0 \pm 2.4$ & 0.132 \\
\hline Delta angle $\left(^{\circ}\right)$ & $86.6 \pm 4.0$ & $87.4 \pm 3.0$ & $86.7 \pm 4.1$ & 0.610 \\
\hline Outlier for femoral component in coronal plane (\%) & 67 & 70.3 & 59.6 & 0.445 \\
\hline Outlier for tibial component in coronal plane (\%) & 14.3 & 12.1 & 12.7 & 0.905 \\
\hline
\end{tabular}

Values are presented as mean \pm standard deviation.

Group A: simultaneous bilateral total knee arthroplasty (SBTKA) on the right knee, Group B: SBTKA on the left knee, Group C: unilateral TKA. 
spective, the debate on SBTKA vs. staged procedure still centers on determination of the most cost-effective method of treatment. In a healthcare system where patients themselves are primary payers of medical services, two separate surgeries increase the total cost of procedure in an incremental manner and are beyond the means of most patients. Our hospital is a welfare trust hospital, which offers international standard yet subsidized services to most needy patients with donations and alms making for the cost difference. Patients usually bear the cost of implant and medication with a subsidy on hospital accommodation, theatre charges and surgeon and anesthetist fees. Thus, the total cost of treatment plays an important role for an individual patient in determining UTKA vs. SBTKA.

Restoration of the coronal mechanical axis of the lower limb to within $3^{\circ}$ of the normal $\left(180^{\circ} \pm 3^{\circ}\right.$ for the lower limb mechanical axis and $90^{\circ} \pm 3^{\circ}$ for the component alignment) during TKA is associated with decreased loosening and greater long-term survival of the prosthesi ${ }^{16}{ }^{16}$. In this study, we used the $\pm 3^{\circ}$ cutoff to determine component outlier and came up with erroneously high rates of femoral component malalignment (as high as 70\%). However, we are fully cognizant of the fact that this is an arbitrary range and there is as yet inconclusive evidence to support the use.

This contradiction with published literature may be explained by anatomical variations in femoral anatomy in our patients. Computer navigation is still not available in our country, even at for-profit tertiary care centers. As most surgeons perform arthroplasty without computer navigation, we use intramedullary femoral jigs to obtain a fixed $5^{\circ}$ or $6^{\circ}$ valgus distal femoral cut based on the intramedullary rod following the distal femoral axis. However, anatomical variations in femoral anatomy, usually present in osteoarthritic knees, will change the angular relationship between the anatomical axis and the mechanical axis of the femur. Thus, the reliability of using a fixed $5^{\circ}$ to $6^{\circ}$ valgus distal femoral cut in all TKAs for restoring a neutral mechanical axis is questionable. Severity of varus or valgus deformity at the knee, femoral bowing, and angular relationships between the condylar slope and the mechanical axis may significantly impact the angular relationship between the distal femoral anatomical axis and the femoral mechanical axis. Due to retrospective nature of this study, we are unable to comment on the effect of these anatomical variations and their effect on component malalignment or mechanical axis deviations.

Mullaji et al. ${ }^{17)}$ found that $43 \%$ of limbs, despite having wellpositioned femoral and tibial components, had malalignment of the mechanical axis. Their results also showed that preoperative varus deformity of more than $20^{\circ}$ and femoral bowing of more than $5^{\circ}$ were associated with increased risk for postoperative mechanical axis malalignment.

Seo et al. ${ }^{11)}$ compared the differences in alignment between two knees where overlapping procedures were done with two teams and one surgeon. They reported that the second TKA, compared to the first TKA, had a higher incidence rate of outlier with regard to the mechanical femorotibial angle ( $16.2 \%$ vs. $9.0 \%)$, more blood loss ( $735 \mathrm{~mL}$ vs. $656 \mathrm{~mL}$ ), and longer operation time (61 minutes vs. 58 minutes), while there were no significant differences in clinical outcomes. In contrast, no significant differences were observed in the incidence rates of outlier with respect to the coronal component placement angle. Possible causes for the results could be surgeon's fatigue and restricted operation field during the second operation with difficulty in complex soft tissue balancing rather than inadequacy of bony cuts and component placement. Contrary to using overlapping procedures as per Seo et al. ${ }^{11)}$, we made the incision on the second knee after wound closure and dressing of the first knee. Matsuda et al. ${ }^{18)}$ found that postoperative varus alignment resulted in lower patient satisfaction. Chowdhry et al. ${ }^{19)}$ found only a weak correlation between coronal limb alignment and functional outcome. Huang et al. ${ }^{20}$ found that the patients had different satisfaction levels with the first and second knees after SBTKA, which was considered related to the order of TKA not to preoperative symptomatic severity of disease.

Similar to our results, Kilincoglu et al. ${ }^{12)}$ reported no statistically significant difference in the component alignment between the SBTKA group and the UTKA group. Nielsen et al. ${ }^{21)}$ found no significant effect of postoperative alignment on the Forgotten Joint Score (FJS). Neither the FJS nor the Oxford Knee Score (OKS) was able to detect a significant difference between the knees. One study showed that $65 \%$ of the patients undergoing bilateral TKA answered equally when asked to provide separate responses for each $\mathrm{knee}^{22)}$.

Our study has some limitations. First of all, the short follow-up in this study limits conclusions regarding the effect of small, although insignificant, angular differences on longevity of the prosthesis. Secondly, we did not investigate the effect of these angular differences on functional outcome which, though important, was not the objective of this study and has been extensively studied in previous reports. Moreover, to maintain homogeneity of the included cases, rheumatoid patients and patients with a valgus deformity, though representing a small percentage of patients, were excluded. For patients with bilateral knee osteoarthritis (in both SBTKA and UTKA subgroups), separate functional assessment of each knee is very challenging. Patients would not be able 
to distinguish differences between the left side and the right side as most commonly used functional assessment scores, including OKS, Knee Society Score (KSS), and Western Ontario and McMaster Universities Osteoarthritis Index (WOMAC) scores, are based on walking distance, stair-climbing, and the use of walking assistance devices $^{11)}$.

\section{Conclusions}

In conclusion, the angular alignment of components was similar between SBTKA and UTKA.

\section{Conflict of Interest}

No potential conflict of interest relevant to this article was reported.

\section{References}

1. Latifi R, Thomsen MG, Kallemose T, Husted H, Troelsen A. Knee awareness and functionality after simultaneous bilateral vs unilateral total knee arthroplasty. World J Orthop. 2016;7:195-201.

2. Haddad B, Khan W, Mehta V, Mbubaegbu C, Qamar A. Bilateral simultaneous total knee arthroplasty: a patientmatched retrospective observational study. Open Orthop J. 2015;9:499-503.

3. Restrepo C, Parvizi J, Dietrich T, Einhorn TA. Safety of simultaneous bilateral total knee arthroplasty. A meta-analysis. J Bone Joint Surg Am. 2007;89:1220-6.

4. Lombardi AV, Mallory TH, Fada RA, Hartman JF, Capps SG, Kefauver CA, Dodds K, Adams JB. Simultaneous bilateral total knee arthroplasties: who decides? Clin Orthop Relat Res. 2001;(392):319-29.

5. Maniar RN, Baviskar JV, Singhi T, Maniar P, Nayak R. Influence of bilateral sequential total knee arthroplasty on functional recovery. Indian J Orthop. 2013;47:23-30.

6. Ekinci Y, Oner M, Karaman I, Kafadar IH, Mutlu M, Argün M. Comparison of simultaneous bilateral with unilateral total knee arthroplasty. Acta Orthop Traumatol Turc. 2014;48: 127-35.

7. Zeni JA Jr, Snyder-Mackler L. Clinical outcomes after simultaneous bilateral total knee arthroplasty: comparison to unilateral total knee arthroplasty and healthy controls. J Arthroplasty. 2010;25:541-6.

8. Lim JB, Chou AC, Chong HC, Lo NN, Chia SL, Tay KJ, Yeo
SJ. Are patients more satisfied and have better functional outcome after bilateral total knee arthroplasty as compared to total hip arthroplasty and unilateral total knee arthroplasty surgery? A two-year follow-up study. Acta Orthop Belg. 2015;81:682-9.

9. Ritter MA, Harty LD, Davis KE, Meding JB, Berend M. Simultaneous bilateral, staged bilateral, and unilateral total knee arthroplasty: a survival analysis. J Bone Joint Surg Am. 2003;85:1532-7.

10. Bagsby D, Pierson JL. Functional outcomes of simultaneous bilateral versus unilateral total knee arthroplasty. Orthopedics. 2015;38:e43-7.

11. Seo JG, Lee BH, Moon YW, Chang MJ, Park SH. Disparate postoperative results in the first and second knees on simultaneous bilateral total knee arthroplasty. J Arthroplasty. 2014;29:2331-6.

12. Kilincoglu V, Unay K, Akan K, Esenkaya I, Poyanli O. Component alignment in simultaneous bilateral or unilateral total knee arthroplasty. Int Orthop. 2011;35:43-6.

13. Azar FM, Canale ST, Beaty JH. Campbell's operative orthopaedics. 13th ed. Philadelphia, PA: Elsevier; 2017. p404.

14. van Jonbergen HP, Scholtes VA, van Kampen A, Poolman RW. A randomised, controlled trial of circumpatellar electrocautery in total knee replacement without patellar resurfacing. J Bone Joint Surg Br. 2011;93:1054-9.

15. Ritter MA, Harty LD. Debate: simultaneous bilateral knee replacements: the outcomes justify its use. Clin Orthop Relat Res. 2004;(428):84-6.

16. Mullaji AB, Marawar SV, Mittal V. A comparison of coronal plane axial femoral relationships in Asian patients with varus osteoarthritic knees and healthy knees. J Arthroplasty. 2009; 24:861-7.

17. Mullaji AB, Shetty GM, Lingaraju AP, Bhayde S. Which factors increase risk of malalignment of the hip-knee-ankle axis in TKA? Clin Orthop Relat Res. 2013;471:134-41.

18. Matsuda S, Kawahara S, Okazaki K, Tashiro Y, Iwamoto Y. Postoperative alignment and ROM affect patient satisfaction after TKA. Clin Orthop Relat Res. 2013;471:127-33.

19. Chowdhry M, Bamne AB, Na YG, Kang YG, Kim TK. Prevalence and predictors of post-operative coronal alignment outliers and their association with the functional outcomes in navigated total knee arthroplasty. J Arthroplasty. 2014;29: 2357-62.

20. Huang S, Li X, Tang Y, Stiphan S, Yan B, He P, Xu D. Different patient satisfaction levels between the first and second knee in the early stage after simultaneous bilateral total knee 
36 Qadir et al. Component Alignment between Simultaneous Bilateral vs. Unilateral TKA

arthroplasty (TKA): a comparison between subjective and objective outcome assessments. J Orthop Surg Res. 2017;12: 121.

21. Nielsen KA, Thomsen MG, Latifi R, Kallemose T, Husted $H$, Troelsen A. Does post-operative knee awareness differ between knees in bilateral simultaneous total knee arthroplasty? Predictors of high or low knee awareness. Knee Surg
Sports Traumatol Arthrosc. 2016;24:3352-8.

22. Nakahara H, Okazaki K, Hamai S, Okamoto S, Kuwashima $\mathrm{U}$, Higaki H, Iwamoto Y. Does knee stability in the coronal plane in extension affect function and outcome after total knee arthroplasty? Knee Surg Sports Traumatol Arthrosc. 2015;23:1693-8. 